\title{
Drag Reduction by Application of Different Shape Designs in a Sport Utility Vehicle
}

\author{
O. Elsayed", A. Omar, A. Jeddi, S. Elhessni and F.Z. Hachimy
}

School of Aerospace and Automotive Engineering, International University of Rabat, LERMA. Campus UIR, Parc Technopolis, Rocade Rabat-Salé, 11100 - Sala Al Jadida - Maroc

Phone: +212530103131

\begin{abstract}
Road vehicles drag is a direct consequence of a large wake area generated behind. This area is created owing to the vehicle shape, which is determined by the class, functional and aesthetic of the vehicle. Aerodynamic characteristics are a ramification and not the reason for the vehicle architecture. To enhance pressure recovery in the wake region, hence reduce drag, three different passive flow control techniques were applied to sport-utility-vehicle (SUV). A threedimensional SUV was designed in CATIA, and a numerical flow simulation was conducted using Ansys-Fluent to evaluate the aerodynamic effectiveness of the proposed flow control approaches. A closed rectangular flap as an add-on device modifies the wake vortex system topology, enhances vortex merging, and increases base pressure which leads to a drag reduction of $15.87 \%$. The perforated roof surface layer was used to delay flow separation. The measured base pressure values indicate a higher-pressure recovery, which globally reflected in a drag reduction of $19.82 \%$. Finally, air guided through side rams was used as steady blowing. A steady passive air jet introduced at the core of the longitudinal trailing vortices leads to a confined wake area. The net effects appear in a global increase in the base pressure values and the pronounced drag reduction of $22.67 \%$.
\end{abstract}

ARTICLE HISTORY

Received: $21^{\text {st }}$ Jan 2021

Revised: 30th June 2021

Accepted: 23 $3^{\text {rd }}$ Aug 2021

\section{KEYWORDS}

Passive flow control;

Perforated roof;

Side ram;

Pressure recovery;

Vortex merging

\section{INTRODUCTION}

One of the main factors that influence vehicle fuel consumption is aerodynamic drag. More than $80 \%$ of the aerodynamic drag is pressure drag, as declared by Wood [1]. Therefore, pressure drag reduction is of great importance in the transportation field. Road vehicle drag reduction has long been initiated; hence numerous approaches were proposed. Flow control is either a passive or active method. Active flow control techniques enable a free shape design. They can alter flow fields without the necessity for external shape changes. Active flow control is essentially applied at points of flow separation to control the early wake formation [2], [3]. Active techniques require extra energy and can be corrected during the control process and thus applied in real-time and unsteady flow control. Passive flow control techniques require no energy, are easy to apply, but cannot be modified during the control process [4], [5].

Passive flow control techniques developed based on flow physics prior knowledge can be of high effectiveness and efficiency. Lee and Ko [6] conducted a study of flow over Gurney-type flaps; they achieved a higher drag reduction value compared to that obtained by solid flaps. Altaf et al. [7] investigated the use of the elliptical flap numerically as an addon rear device to reduce the drag of a TXG MAN long haul truck, where the maximum drag reduction of $11.1 \%$ was achieved. Beaudoin and Aider [8] experimentally studied a 3D bluff body with flaps added at the edges. Their results showed that the two flaps on the rear side edges reduce the drag by $25 \%$. Ha, et al. [9] used both experimental and computational approaches to study the effect of using a rear downward flap applied to a pickup truck. Their study reveals that the drag reduction is proportional to the flap length. Kim et al. [10] have studied experimentally and numerically the case of different cab-roof fairing CRF models added to a heavy vehicle. The base CRF proved to achieve a $15 \%$ reduction of drag, and additional modifications to the CRF increases the drag reduction up to $3.62 \%$. Jie et al. [11] carried out a numerical investigation of two flap structures used on Ahmed body models at $25^{\circ}$ and $35^{\circ}$. Up to $21.2 \%$ drag reduction was achieved.

Paul et al. [12] carried out a computational and experimental study for a vane-type vortex generator array and spoiler added to the boot side of a passenger car. The implementation of vortex generators reduces the drag increased by the crosswind up to $23 \%$ and achieves up to $11.5 \%$ reduction in fuel consumption using both techniques. Mariotti et al. [13] studied numerically contoured grooves and their impact on drag reduction by delaying separation. Contoured grooves on boat tail lateral surfaces significantly influence the separation region, and the results show that up to $9.7 \%$ drag reduction can be achieved. Sadeghipour et al. [14] studied the contribution of porous media in passive flow control, and LoriteDiez et al. [15] investigated the contribution of rear cavities in reducing drag up to $10 \%$. Many researchers studied the effect of the surface roughness using dimples or perforation on the drag forces and stability of the vehicles where early boundary layer transition to turbulent was observed. Mode [16] used dimples on the flat plate to prevent boundary layer separation and reduce the wake. Altaf [7] reported a reduced and diluted wake back of the truck by using spherical dimples placed on the cab of the truck. Wong and Del [17] and Shaw et al. [18] concluded that introducing surface dimples to the road vehicles resulted in drag reduction and improving downforce and stability. 
The active flow control methods are also of great importance as they have a significant impact on drag reduction without affecting the shape of the car and its habitable volume. The implemented solutions generally consist of suction and blowing. Brunn et al. [19] investigated the flow field over Ahmed body modal using slant angles of $25^{\circ}$ and $35^{\circ}$ as two configurations. For the $25^{\circ}$ case, steady blowing in the vertical direction was used. They claimed that wake vorticity could be reduced with a drag reduction of $2.5 \%$. Heinemann et al. [20] applied steady blowing to a real car modal to observe its impact on the lift and drag at the rear part. They applied continuous slots at various positions above and beneath the rear window of the car. Laser Doppler Anemometry (LDA) was used to measure the rear flow field velocity. The results reveal that the active flow control reduces the lift in the rear axle with small losses in drag performance. Littlewood and Passmore [21] applied steady blowing at a variety of angles on the roof trailing edge of a $1 / 4$ square back vehicle. They used direct force measurement, hot wire anemometry (CTA), and particle image velocimetry (PIV) to investigate the impact of steady blowing on the vehicle wake structure. The results have shown that the intended benefits were attained, but the large mass flow rate required limits the use of the technique in road vehicles. Aubrun et al. [22] conducted a study on Ahmed's body modal with a slant angle of 25 . Steady blowing microjets were applied at 6 mm downstream of the separation line. They observed the influence of this active control method on the aerodynamic drag. The results have shown that the drag was reduced by 9 to $14 \%$, depending on the Reynolds number.

Zhang et al. [23] studied four individual actuations produced by steady blowing on the rear edges of an Ahmed body model. The results have shown that up to $29 \%$ drag reduction can be achieved for the combination of the four actuations. Cerutti et al. [24] carried out an experimental investigation of continuous blowing for a square back vehicle model. They mounted four rectangular blowing jets to the rear part of a van model, and they were able to reach a maximum of $12 \%$ drag reduction. Harinaldi and Tarakka [25] conducted research using both computational and experimental methods. They applied blowing techniques on a bluff body van model rear part. The bluff body van model is modified based on the Ahmed model. They have reported a drag reduction of $11.11 \%$. Harinaldi et al. [26] studied a van body equipped with suction at the rear part. They concluded that active flow control could reduce the aerodynamic drag by $16.32 \%$. Jahanmiri and Abbaspour [27] investigated the application of an active flow control method to Ahmed body modal with a $35^{\circ}$ rear slant angle. They used suction in the boundary layer to delay separation; it is positioned at the beginning of the rear slant surface and the blowing at the middle of the rear vertical part of the modal. Baek and Lee [28] conducted an analysis of continuous blowing used for a realistic vehicle model. They found that the effect of continuous blowing increased with Reynolds number (Re). The maximum drag reduction achieved was about 7.5\%. Mosiezny et al. [29] studied numerically the case of an active flow control device based on the Coanda effect added to a tractor-trailer. The results show that the drag can be reduced up to $11 \%$.

Passive flow control on a generic car model was experimentally studied by Fourrié et al. [30]. A deflector positioned on the upper edge of the model rear window was used by Chainani and Perera [31]. They have performed a wind tunnel measurement at two different Reynolds numbers. Measurement of flow properties indicates a drag reduction of $9 \%$ for different deflector angles. The results showed a large separation was produced, and it interrupts the counter-rotating longitudinal vortices. A road vehicle is a bluff body of a complex geometry run in proximity to the ground with a 3-D separated flow developed. In this work, a numerical prediction of the flow structure for SUV, base-line, and with three different modified geometry designs were investigated. Results obtained will help a better understanding of this complex problem and will further enable reducing the dominated pressure drag over the SUV.

\section{MODELS}

The model used for this study is based on a general Sport Utility Vehicle (SUV) shape, as shown in Figure 1. The model geometry was designed by CATIA and was imported to Ansys 17.2 for the numerical simulation. This model was considered a baseline case, and it was used as a benchmark for the modified cases by add-on devices.

\section{Closed Rectangular Flap Case}

The flow over the front vehicle parts of a sub-sonically moving body through the air is manageable. In contrast to that over the rear. The slant angle $\varphi$ (measured from the horizontal line to the vehicle rear surface inclination) below $15^{\circ}$ results in a minimum net drag value [32]. The closed rectangular flap was added to the SUV rear roof end to reduce the pressure drag. The geometry of the closed rectangular flap is shown in Figure 2. As an attempt to improve the rectangular flap design, the sides were closed.

\section{Modified Roof Surface with Cavities}

Five rows of perforation were designed. The modified roof surface with cavities and their respective dimensions is shown in Figure 3. Perforations were placed on the top roof at the point of flow separation to generate a favourable circulation. The circulation enhances the mixing of the flow and re-energises the boundary layer. This will delay the flow separation [7].

\section{Side Rams}

Side-rams were designed with an internally curved duct and positioned carefully at the vehicle sides to introduce a steady passive jet into the wake region. Tangential velocity increases toward the vortex core, causing base pressure to drop. Steady jet favourably interacts with the trailing edge vortices and decreases the total net drag. The SUV with the side-rams dimensions is shown in Figure 4. 

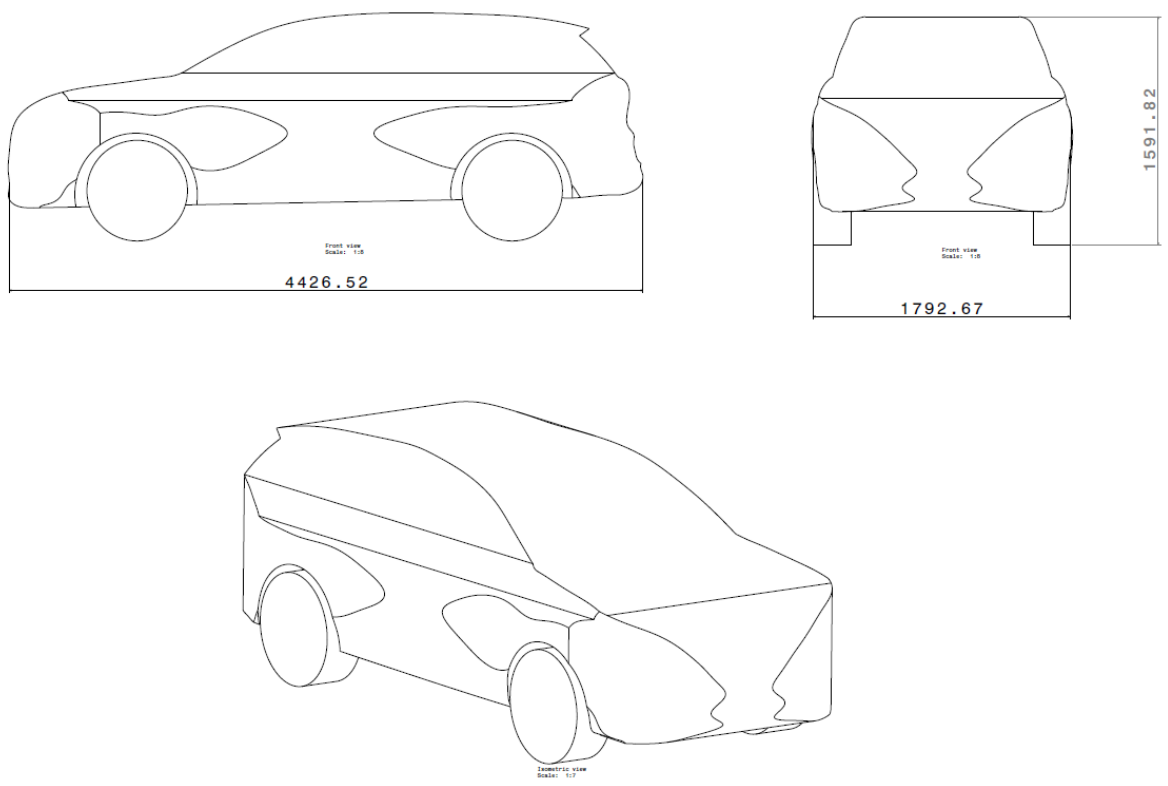

Figure 1. SUV simplified model geometry.
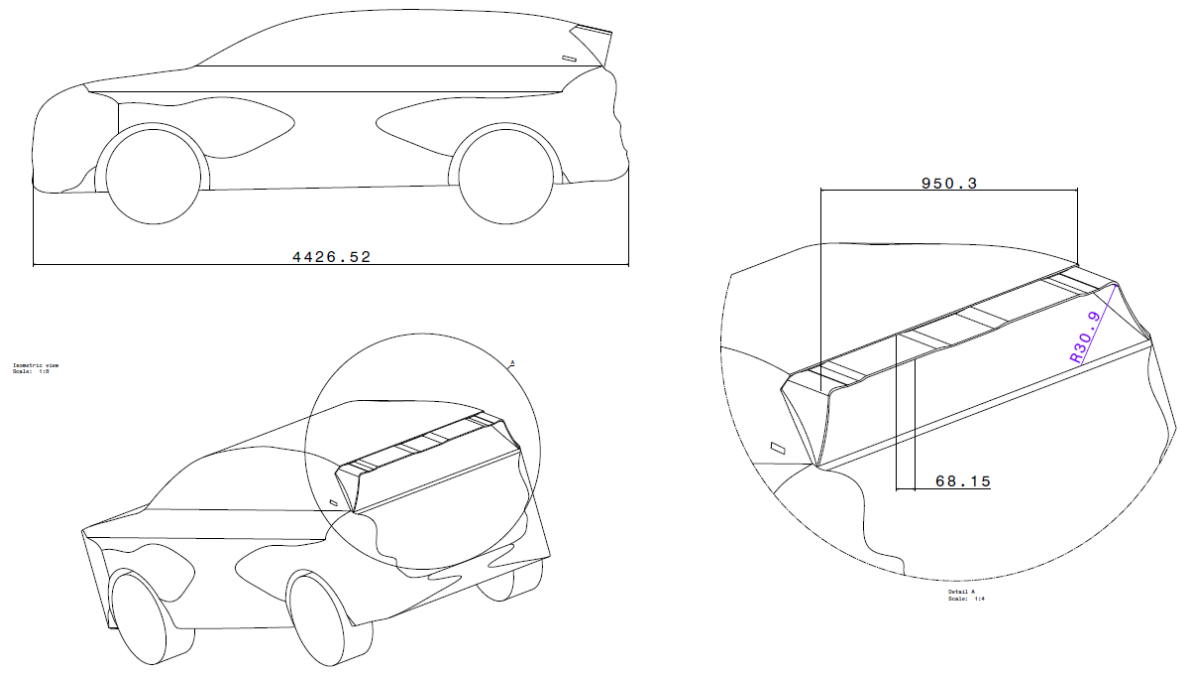

Figure 2. Closed rectangular flap geometry.

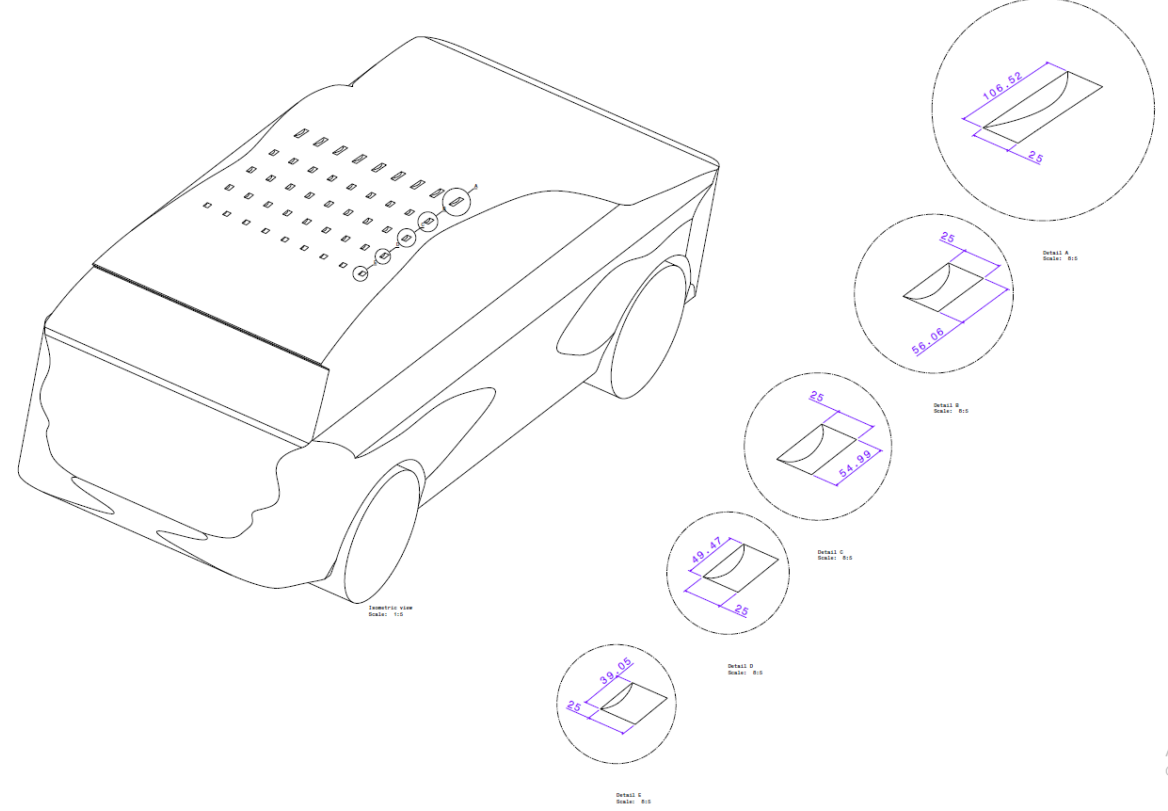

Figure 3. Modified roof surface with rows of cavities. 

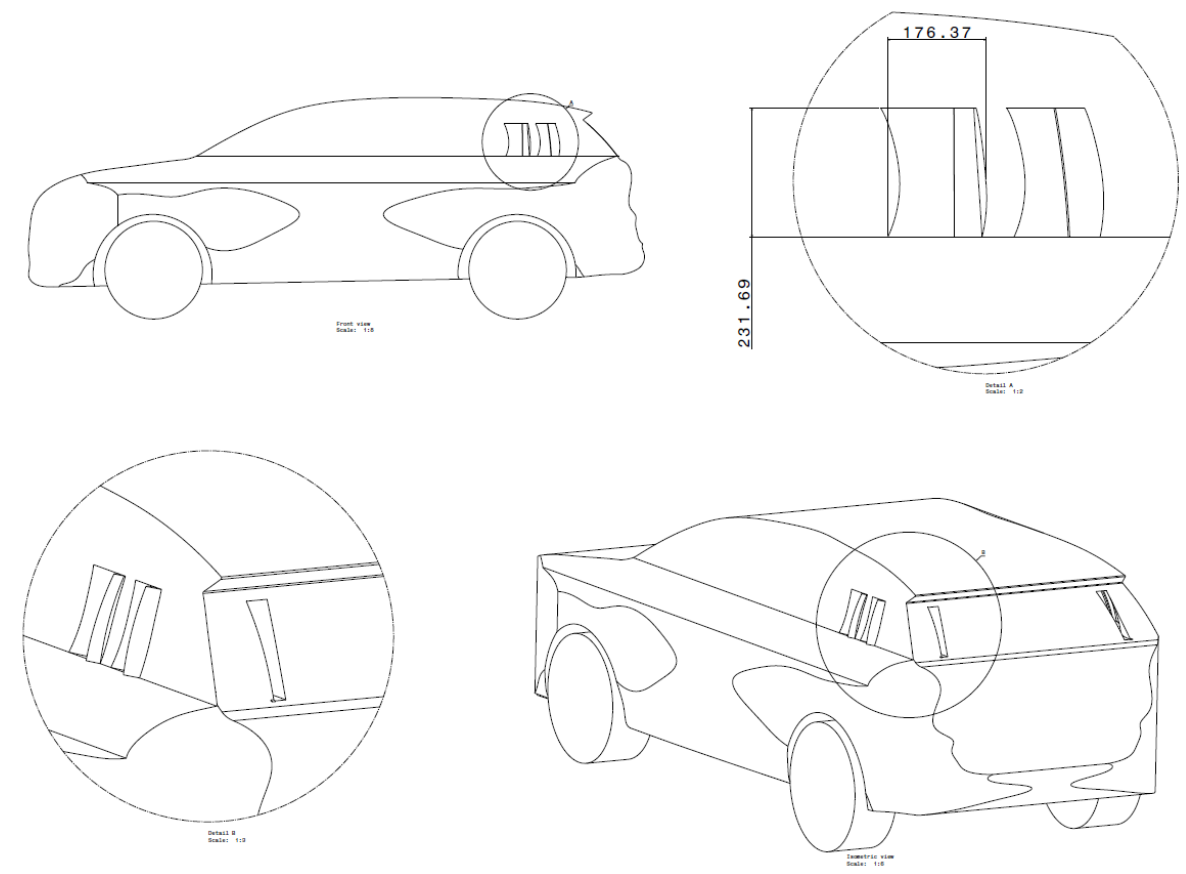

Figure 4. Side-ram model.

\section{NUMERICAL SIMULATION}

\section{Meshing and Physical Setup}

Using the mesh module of the Ansys workbench, proper meshes were generated over the flow domain of (X, Y, Z, $\mathrm{X},-\mathrm{Y},-\mathrm{Z}$ ) coordinates equal to $8,8,8,15,8,0 \mathrm{~m}$, respectively. The coordinates presenting the proper dimension of the encloser for similar applications [7], [11]. The values and parameters of the mesh generated are tabulated in Table 1. Flow resolution over critical domains and complex geometries were carefully treated by generating enough mesh numbers. To resolve the viscous flow region for the SUV model, the $\mathrm{Y}^{+}$value of 30 was set [11], where the minimum spacing of 1.25e5 was used. The mesh generated over SUV is shown in Figure 5 with the boundary conditions specified at the respective flow domains. Grids of a tetrahedral type were generated on the flow domain and in the proximity of the SUV. Further, the mesh is converted to a polyhedral mesh type due to its faster convergence with fewer iterations and lower residual values and faster solution runtimes. Mesh visualisation of the polyhedral type around the SUV in a close view is shown in Figure 6. The sample of the residual history shown in Figure 7 proves the quality of the mesh and the stability of the code.

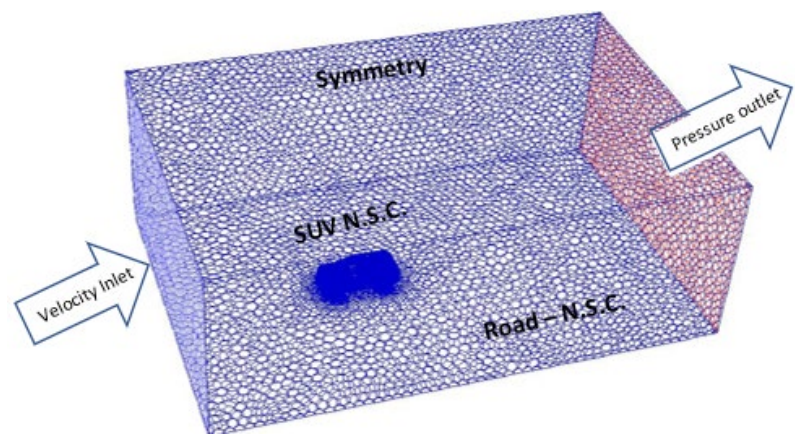

Figure 5. Fluid flow domain and boundary conditions.

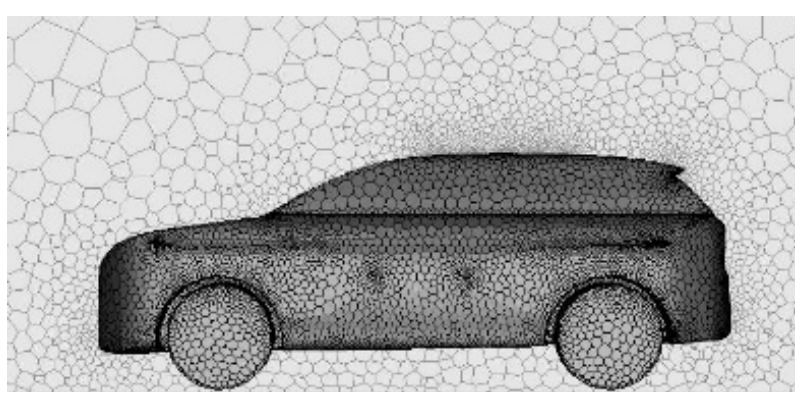

Figure 6. Mesh around SUV close view. 


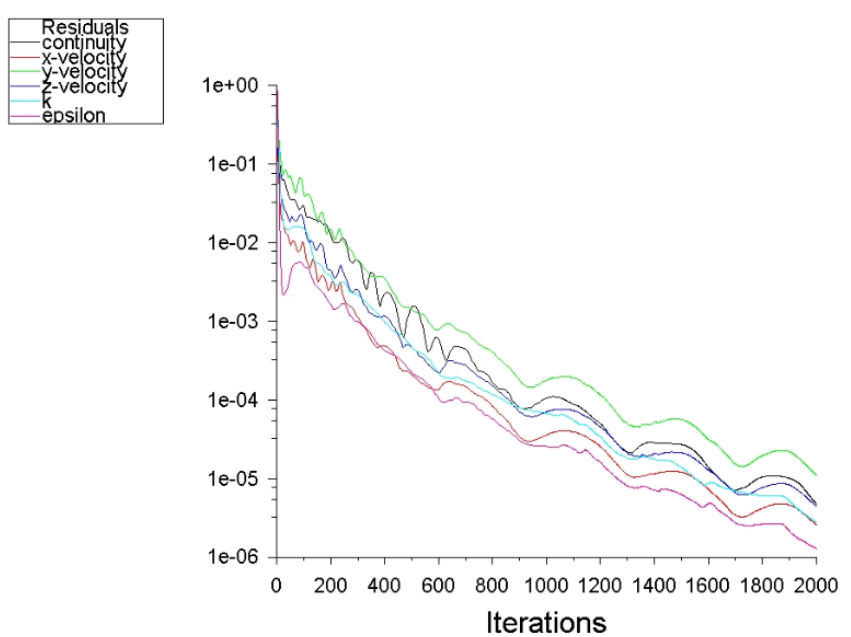

Figure 7. Residual history for cavity case.

\section{CFD Model Validation}

A basic ground vehicle type of bluff body was studied experimentally by Ahmed et al. [33], details of the wake structure, pressure measurements, wake survey, and force measurements were evaluated. This model is widely used as a validation and benchmark tool for the different numerical setups of road vehicle flow analysis [7, 11 and 32$]$. In this study, Ahmed body of a geometry shown in Figure 8 and 9 was used as a validation tool. The flow speed and the angle of the slant at the rear of the body were taken as $\mathrm{V}_{\infty}=60 \mathrm{~m} / \mathrm{s}$ and $\varphi=25^{\circ}$, respectively. Reynolds number based on a model length is $4.29 \times 10^{6}$. Details of mesh generated over the flow domain and the physical solver parameters used for the flow solution are shown in Table 1. A close view of the mesh generated around Ahmed's body is shown in Figure 8.

Table 1. Mesh and solver parameters.

\begin{tabular}{|c|c|c|c|}
\hline & & \multicolumn{2}{|c|}{ Parameter } \\
\hline & & Ahmed Body & SUV \\
\hline Volume mesh & & \multicolumn{2}{|c|}{ polyhedral } \\
\hline \multirow[t]{9}{*}{ Boundary layer } & Inflation option & \multicolumn{2}{|c|}{ First aspect ratio 5} \\
\hline & First aspect ratio & \multicolumn{2}{|c|}{5} \\
\hline & Maximum layer & \multicolumn{2}{|c|}{15} \\
\hline & Growth rate & \multicolumn{2}{|c|}{1.2} \\
\hline & Minimum face size & $5.0 \mathrm{e}-4 \mathrm{~m}$ & $3.16 \mathrm{e}-4 \mathrm{~m}$ \\
\hline & Minimum Edge length & $8.8 \mathrm{e}-2 \mathrm{~m}$ & $1.55 \mathrm{e}-3 \mathrm{~m}$ \\
\hline & Max face size & $0.512 \mathrm{~m}$ & $0.995 \mathrm{~m}$ \\
\hline & Total no. of nodes & 199773 & 2586940 \\
\hline & Total no. of elements & 1017640 & 1814760 \\
\hline Physical properties & Flow characteristics & \multirow{3}{*}{\multicolumn{2}{|c|}{$\begin{array}{c}\text { Steady, incompressible, coupled, density-based RANS [34] } \\
\text { K-epsilon Realizable [35] } \\
\text { Non-equilibrium wall functions }\end{array}$}} \\
\hline \multirow[t]{3}{*}{ Turbulence model } & Model & & \\
\hline & Near wall treatment & & \\
\hline & Flow velocity & $60 \mathrm{~m} / \mathrm{s}$ & $35 \mathrm{~m} / \mathrm{s}$ \\
\hline
\end{tabular}
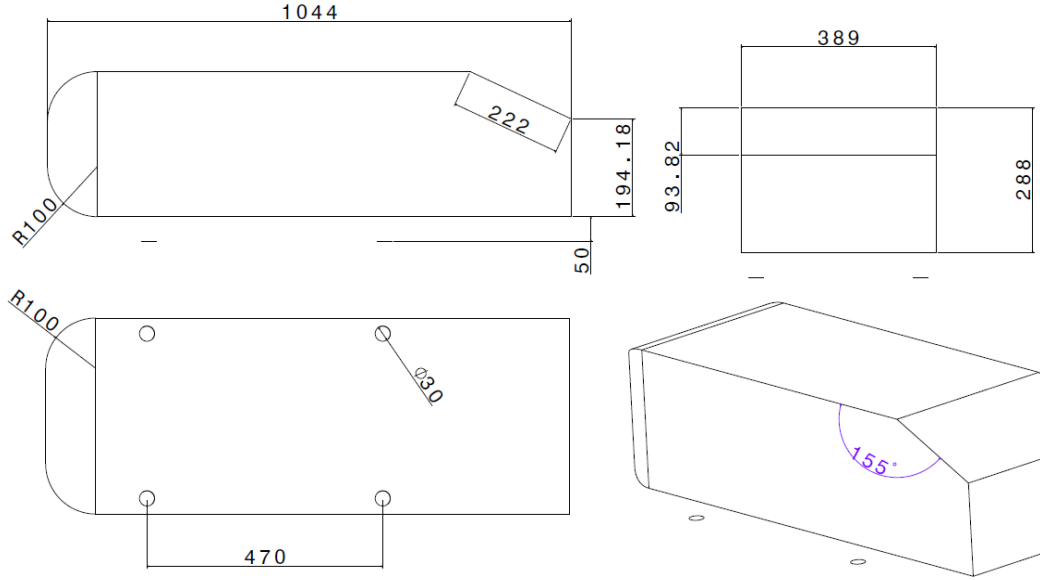

Figure 8. Dimension of Ahmed body [33]. 


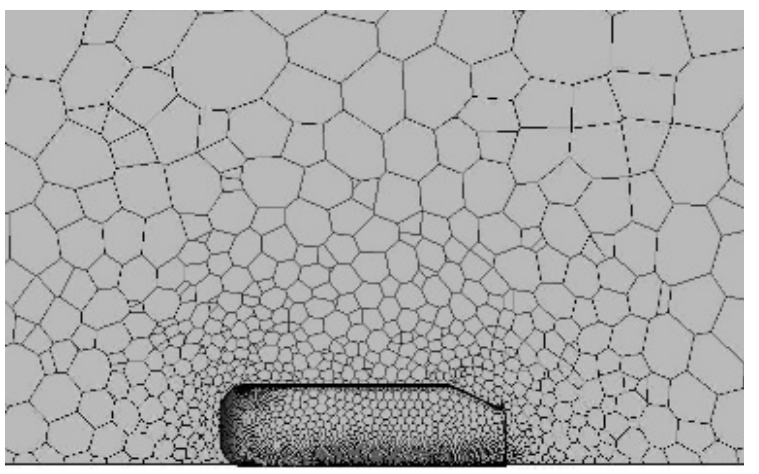

Figure 9. Mesh visualisation around Ahmed body edges.

\section{Pressure contours and streamlines distribution}

The pressure contour over Ahmed body is shown in Figure 10(a); streamlines distribution reveals a shear layer rolls up into two rotating flow regions situated one over another, as shown in Figure 10(b). The crossflow velocity vector distributions at two vertical planes behind Ahmed's body are shown in Figure 11 (a) and 11(b). A strong pair of vortices at the side edges of the slant surface and at the lower edges of the body are indicated, as seen in Figure 11(a). The shear layer, coming off the slant side edge, rolls up into a two main longitudinal vortex, as in Figure 11(b). The longitudinal trailing vortices decay at a downstream distance of approximately one body length. The numerically predicted net drag coefficient of Ahmed-body is in excellent agreement with the reported net drag coefficient by Ahmed et al. [33]. The result is shown in Table 2, which indicates $87 \%$ of the drag is pressure drag while $13 \%$ is due to viscous effect, which also in very good agreement with the reported value of $85 \%$.

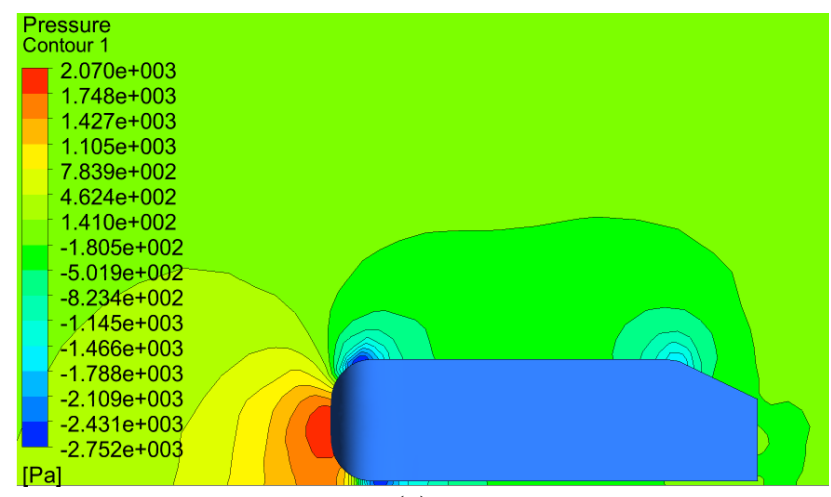

(a)

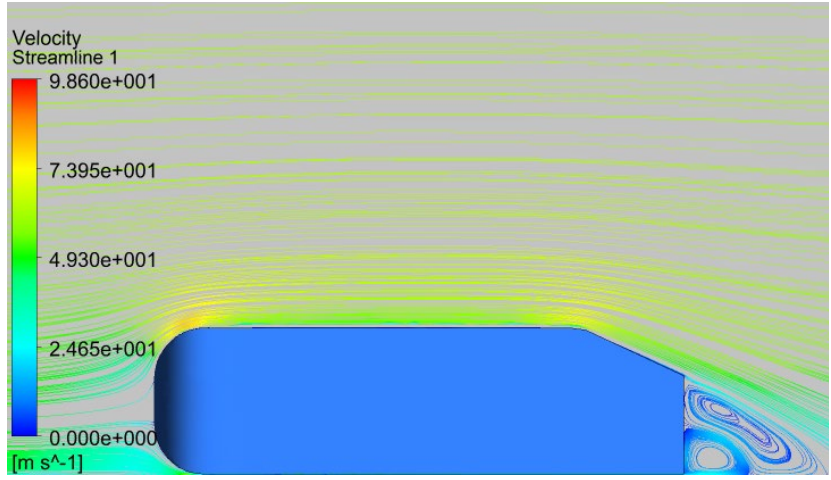

(b)

Figure 10. (a) Pressure contours for $\mathrm{Re}, 1=4.29 \times 106$ at $\mathrm{x} / \mathrm{w}=0.288$ and (b) streamlines distribution for $\mathrm{R}_{\mathrm{e}, \mathrm{l}}=4.29 \times 10^{6}$ at $\mathrm{x} / \mathrm{w}=0.288$ over Ahmed body.

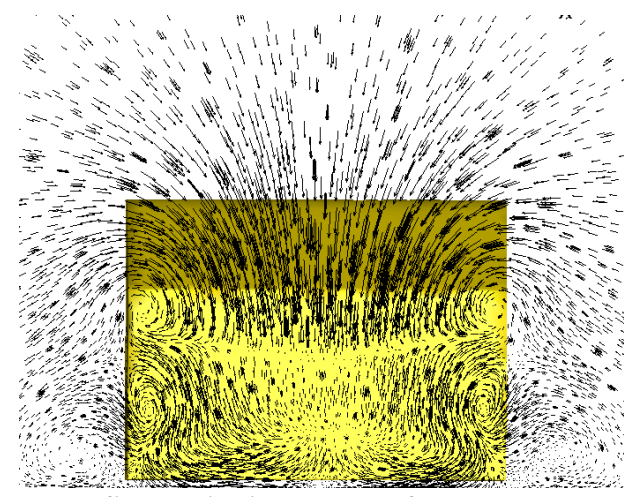

(a) Cross- flow velocity vectors for $\mathrm{R}_{\mathrm{e}, 1}=10.6 \times 10^{6}$ at $\mathrm{z} / 1=0.04$

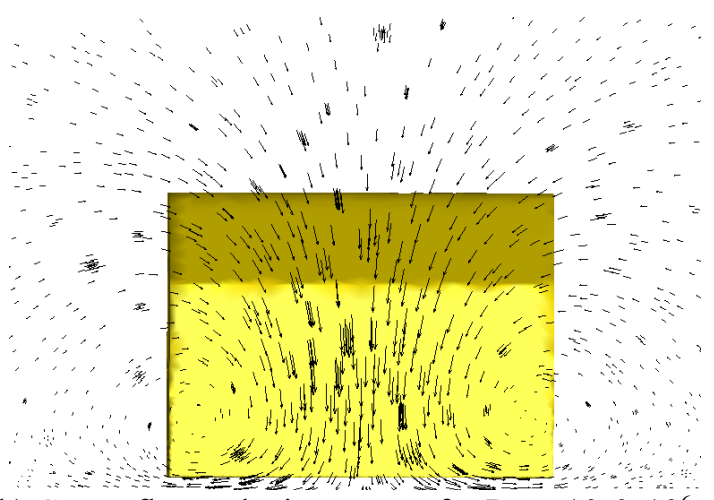

(b) Cross- flow velocity vectors for $\mathrm{R}_{\mathrm{e}, 1}=10.6 \times 10^{6}$ at $\mathrm{z} / \mathrm{l}=0.24$.

Figure 11. Cross-flow velocity vector distributions at the downstream.

Table 2. Drag coefficient results at $\mathrm{R}_{\mathrm{e}}=4.29 \times 10^{6}$ and $\varphi=25^{\circ}$.

\begin{tabular}{lccc}
\hline Model & \multicolumn{3}{c}{ Drag coefficient } \\
\hline Ahmed 1984 & 0.2875 \\
Ansys-Fluent (K-epsilon Realizable) & $\mathrm{C}_{\mathrm{D}, \mathrm{p}}$ & $\mathrm{C}_{\mathrm{D}, \mathrm{f}}$ & $\mathrm{C}_{\mathrm{D}}$ \\
& 0.247 & 0.036 & 0.283 \\
\hline
\end{tabular}




\section{RESULTS AND DISCUSSION}

Road vehicle is bluff bodies in proximity to the ground. Their detailed geometry is complex and internal cavities communicate freely with the external flow. The flow is fully 3-D, boundary layers are turbulent, and flow separation is common. Large turbulent wakes are formed at the rear and contain longitudinal trailing vortices [32]. Those geometric and flow complexities made the road vehicle flow analysis a challenging problem. All the tested cases were performed for a vehicle speed of $35 \mathrm{~m} / \mathrm{s}$, which is equivalent to the upper-speed limit of the highway. No significant change of the flow behaviour is expected for vehicle speed below $35 \mathrm{~m} / \mathrm{s}$ since it is still under the incompressible flow speed range.

\section{Surface Pressure and Shear Stress}

Direct evaluation of pressure and skin friction drag through experimental tools is very difficult. Adequately validated CFD codes can contribute greatly to obtain detailed pressure and surface shear stress distributions which are reflected directly in a better understanding of vehicle aerodynamics. In Figure 12(a) and 12(b), the numerically predicted pressure and the skin friction distribution over SUV are shown, from which a direct net vehicle drag calculation is possible.

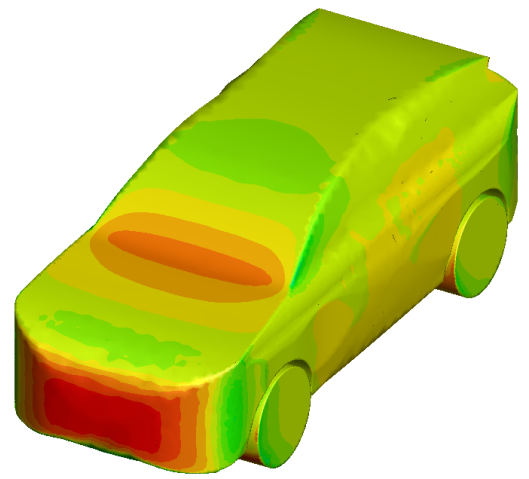

(a)

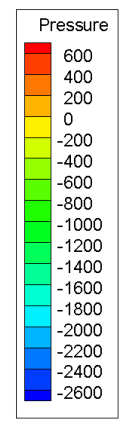

ure conto

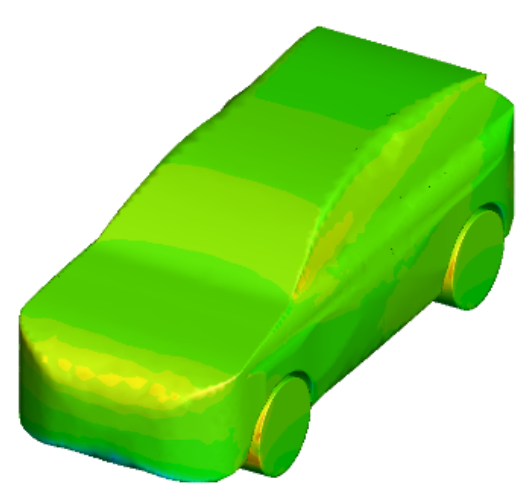

(b)

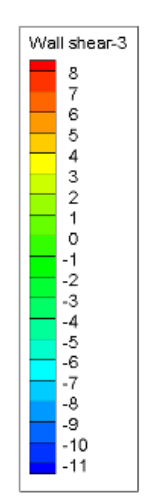

\section{Velocity Distributions, Base Pressure and Drag Evaluation}

Streamwise trailing vortices dominate the road vehicle wake; it originates from a different region on the vehicle body. They are the main drag producer; hence drag is minimised by minimising vortex drag.

\section{Baseline}

SUV wake flow details are shown in Figure 13 (a) to 13(d). Streamlines distributions are shown in Figure 13(a). It reveals a large wake region of a typical bluff body with three different vortices. The vortices were generated at the rear top roof-wind shield juncture and at the two edges of the rear bumper. The last two are two small counter-rotating vortices. A large separation bubble was generated due to the vehicle under-side shear layer separation on the ground. The vortex system topology confirms the Ahmed et al. [33] finding for the square-back vehicle.

The cross-velocity vector diagrams in the near wakefield are shown in Figure 13(b) and 13(c). Cross velocity vector distributions clearly indicate the three vortices and confirm the earlier observations. The system of vortices merges with the vehicle bottom shear-layer and roll-up into two main counter-rotating longitudinal trailing vortices, as seen in Figure 13(c). The two small counter-rotating vortices merge into the main longitudinal vortices, and the cross-velocity vectors showed longitudinal vortices decay at the $\mathrm{x} / \mathrm{l}=0.68$.

Base pressure contours are shown in Figure 13(d). The base pressure contour indicates a large region of suction on the lower half of the base surface. While on the upper half pressure counters indicates a mild pressure variation with a gradual increase towards the roof trailing edges. The 3-D drag coefficient was evaluated for the baseline case and found $\mathrm{C}_{\mathrm{D}}=0.397$ using Eq. (1).

$$
C_{D}=D /\left(\rho / 2 V_{\infty}^{2} A_{f}\right)
$$

where, $A_{f}$ is frontal area, and $\rho$ is air density. The summary of the drag values for different SUV cases and their respective drag reduction values are tabulated in Table 3.

\section{Closed rectangular flap}

The major aerodynamics problem of road vehicles as a bluff body is at the rear; the flow over and from the upper rear surface of the vehicle is particularly interesting. Trailing vortices are formed at the lateral edges of slanted surfaces. To restructure the rear vortex system favourably, a closed rectangular flap was used as an add-on device. Streamlines, velocity vectors, and pressure distributions are shown in Figure 14(a) to 14(d). That streamline distributions emphasise the hypothesis of restructuring the wake vortex system. The wake region is stretched compared to that of the baseline case, as indicated in Figure 14(a). The main vortices generated at the upper base surface half as well as the two small counter-rotating vortices formed at the rear bumper edges quickly rolled up and merged, resulting in a pair of coherent 
two counter-rotating longitudinal vortices. The modifications in the vortex system topology enhanced a faster merging and decay of the vehicle wake vortex system.

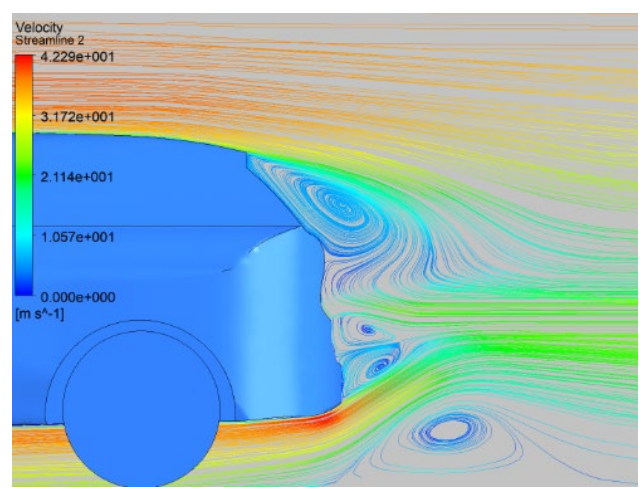

(a) Streamlines at $\mathrm{y} / \mathrm{w}=0.12$

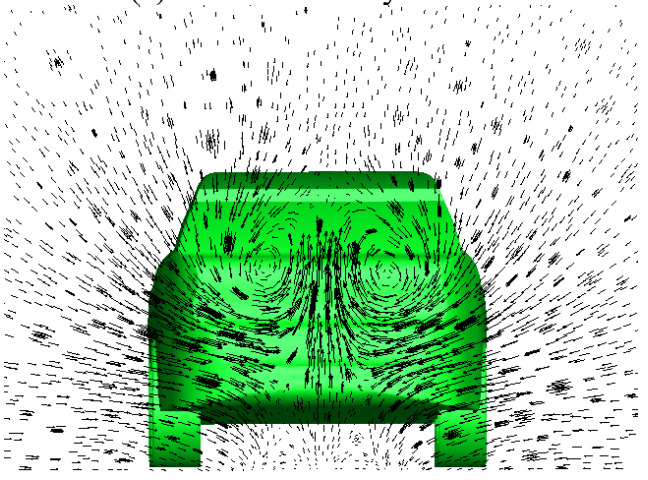

(c) Velocity vectors at $\mathrm{x} / \mathrm{l}=0.2$

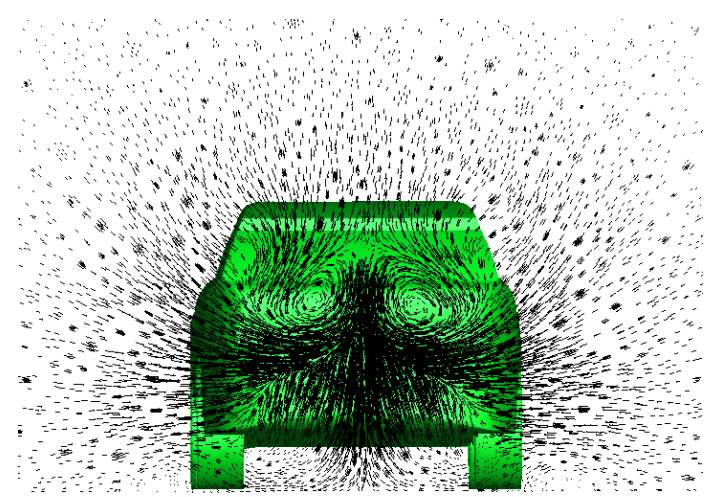

(b) Velocity vectors at $\mathrm{x} / \mathrm{l}=0.04$

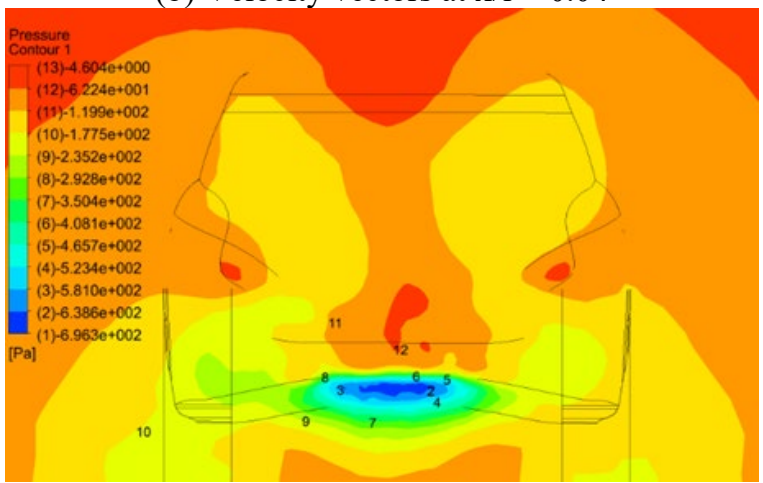

(d) Pressure contours at $\mathrm{x} / \mathrm{l}=0.004$

Figure 13. Base-line case, streamlines, velocity vectors and pressure contour distributions at $R_{e, 1}=10.6 \times 10^{6}$.

SUV wake surveys are shown in Figure 14(b) to 14(c). Cross-velocity vectors distribution at $\mathrm{x} / 1=0.04$ indicates a pair of counter-rotating vortices, Figure 14 (b). This vortex pair roll-up and merge into a single pair at $\mathrm{x} / \mathrm{l}=0.2$, as shown in Figure 14(c). The base pressure contours are shown in Figure 14(d). It indicates a large region of suction on the lower half of the base surface comparable to that shown in the baseline configuration case. While on the upper half, the base pressure increased gradually towards the closed-rectangular flap edges and showed a relatively uniform distribution across the flap width, indicating the $2 \mathrm{D}$ behaviour of the flow over this area. The drag coefficient is found to be reduced to the value of $\mathrm{C}_{\mathrm{D}}=0.334$, with a $15.87 \%$ reduction compared to the baseline case drag value.

\section{Modified roof surface with cavities}

Roof surface cavities were selected as a passive flow control means. A combination of two sets of cavities was designed and implemented on the SUV roof surface starting at the flow separation point. The hypothesis of this approach is to generate small vortices at the boundary layer zone, which in turn re-energise the turbulent boundary layer. Although a higher skin friction drag is expected, a delay of separation and control of wake formation is essential for net drag reduction.

The confined SUV wake area compared to baseline cases was evidence, as shown in Figure 15. The wake vortex system topology is of a conventional type. Cross velocity vector distributions are shown in Figure 15 (a) to 15(b) reveals an outer lateral movement of the rear roof-wind shield juncture vortex pair. In Figure 15(d), the base surface pressure contours show two regions of suction formed at the lateral sides in the upper surface half. The base pressure values indicate a higher pressure recovery compared to the baseline configuration. This increase in base pressure value is reflected in the net drag reduction of $19.82 \%$ compared to the baseline case drag value (in Table 3 ). 


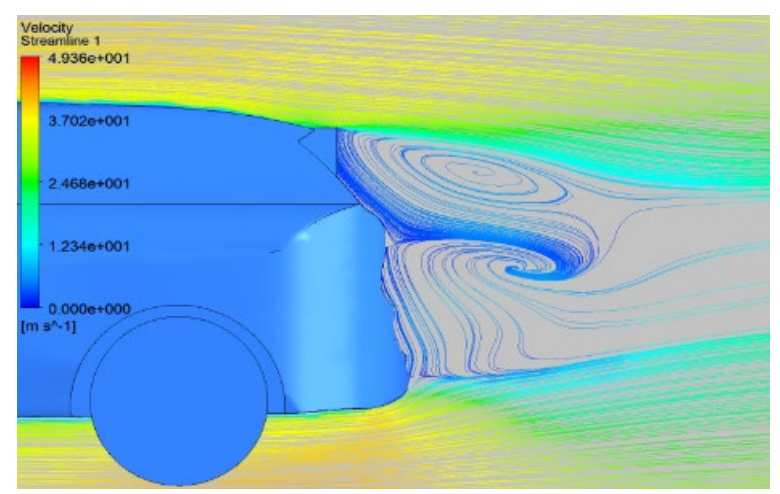

(a) Streamlines at $\mathrm{y} / \mathrm{w}=0.12$

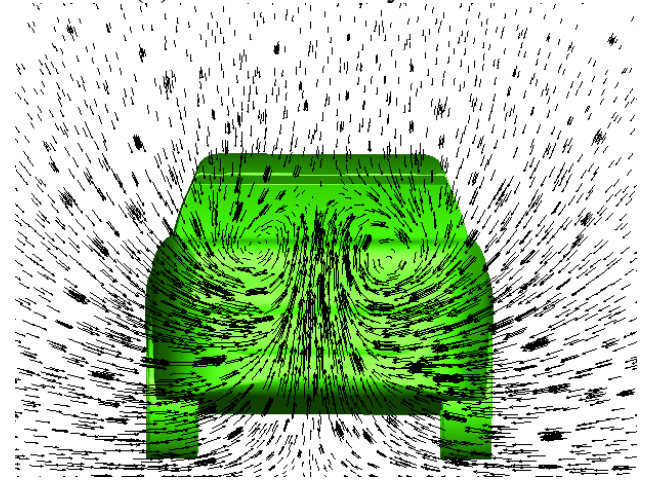

(c) Velocity vectors at $\mathrm{x} / \mathrm{l}=0.2$

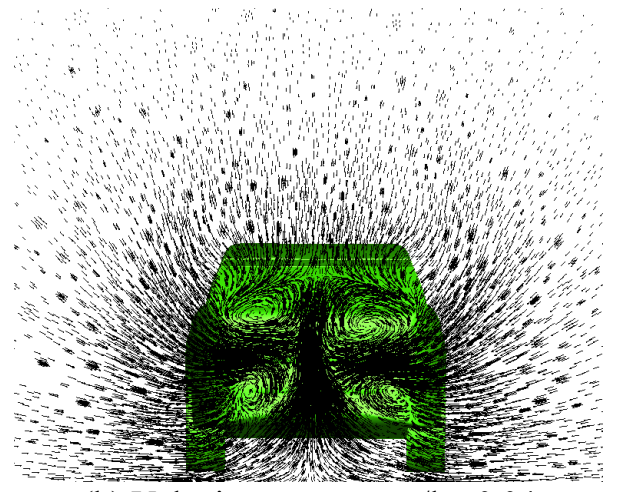

(b) Velocity vectors at $\mathrm{x} / \mathrm{l}=0.04$

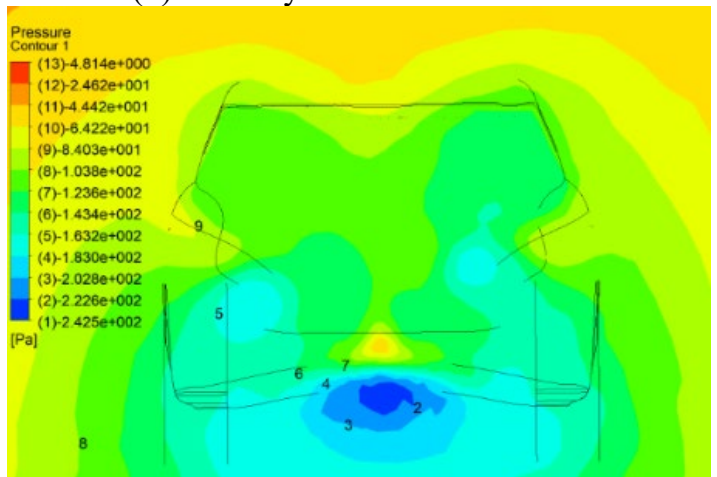

(d) Pressure contours at $\mathrm{x} / \mathrm{l}=0.004$

Figure 14. Closed rectangular flap, streamlines, velocity vectors and pressure contour distributions at $R_{e, 1}=10.6 \times 10^{6}$.

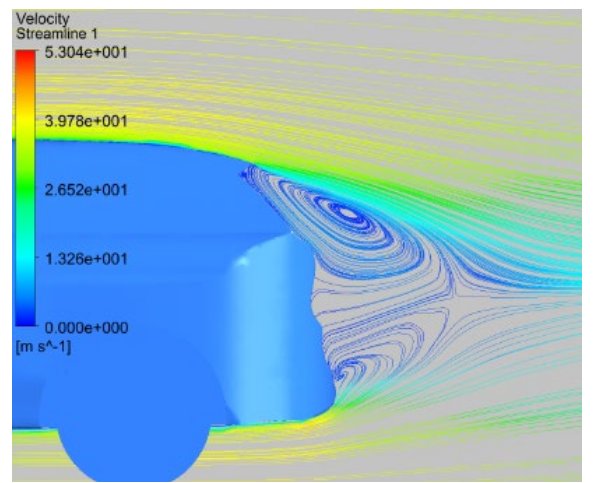

(a) Streamline at $\mathrm{y} / \mathrm{w}=0.12$

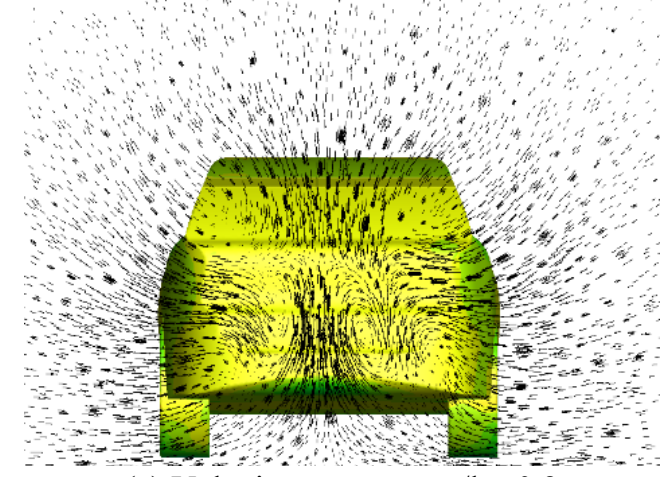

(c) Velocity vectors at $\mathrm{x} / \mathrm{l}=0.2$

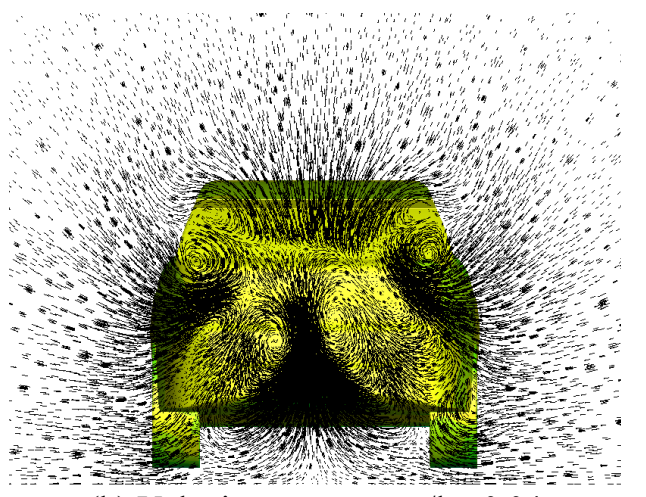

(b) Velocity vectors at $\mathrm{x} / \mathrm{l}=0.04$

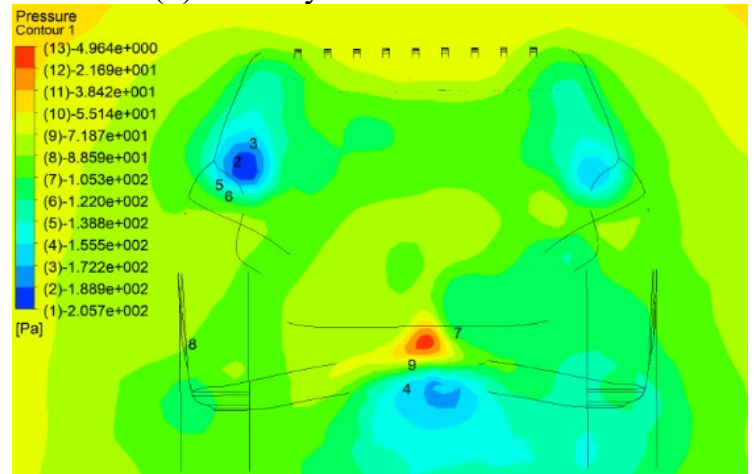

(d) Pressure contours at $\mathrm{x} / \mathrm{l}=0.004$

Figure 15. Modified roof surface with cavities, velocity vectors and pressure contour distributions at $R_{e, 1}=10.6 \times 10^{6}$.

\section{Side-ram}

The aerodynamic drag of the square-back vehicle class is mainly generated due to the loss of pressure in the wake area. The dominant structure is the lower recirculating vortex that will interact with the model base creating a lower base pressure. Air guided through the carefully designed internally curved side rams will act as a passive blowing technique. Steady air is introduced at the area close to the vortex cores. Streamlines distribution at the wake region reveals a confined wake area with a counter-rotating vortex pair, see Figure 15(a). This wake reduced size compared to the baseline case is due to both blowing air from the side rams and the downwards deflecting shear layer, as indicated in Figure 16(b) and 
16(c). The net effects appear in a global increase of the base pressure values are shown in Figure 16(d), and the pronounced drag reduction of $22.67 \%$ compared to the baseline drag value as tabulated in Table 3 .

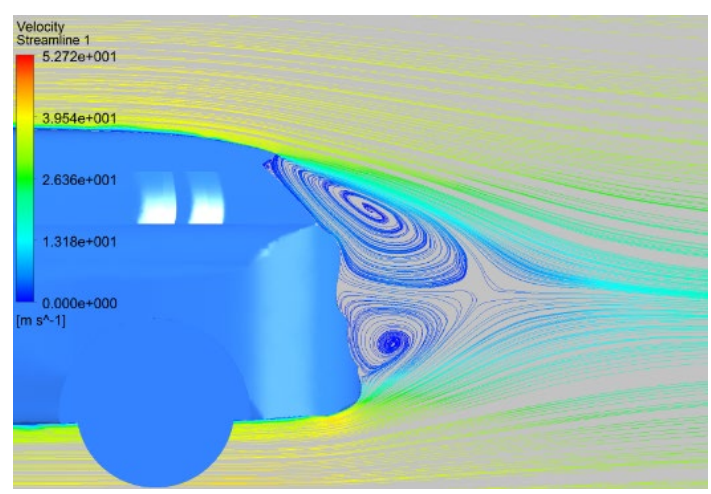

(a) Streamlines at $\mathrm{y} / \mathrm{w}=0.12$

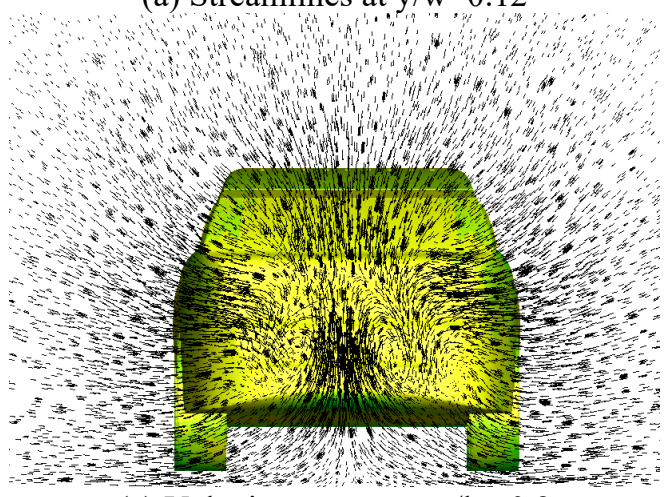

(c) Velocity vectors at $\mathrm{x} / \mathrm{l}=0.2$

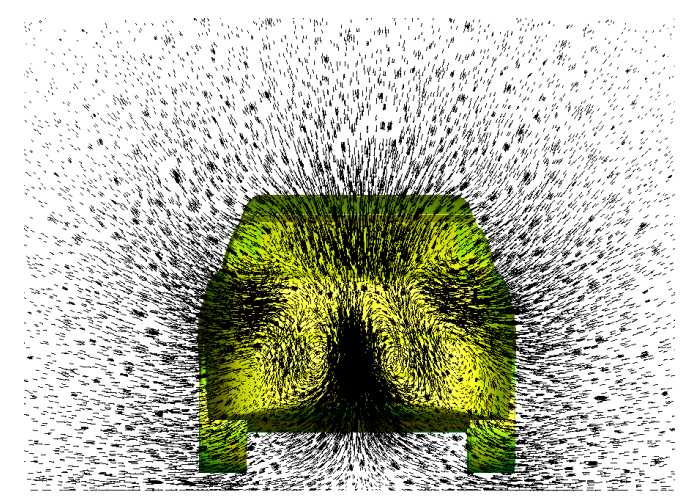

(b) Velocity vectors at $\mathrm{x} / \mathrm{l}=0.04$

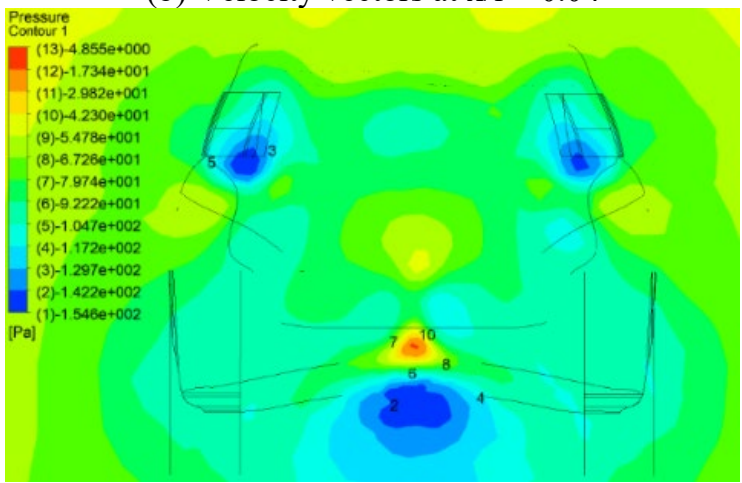

(d) Pressure contours at $\mathrm{x} / \mathrm{l}=0.004$

Figure 16. Side-rams, velocity vectors and pressure contour distributions at $R_{e, 1}=10.6 \times 10^{6}$.

Table 3. Summary of the drag reduction.

\begin{tabular}{lcccc}
\hline SUV case & $C_{D, p}$ & $C_{D, f}$ & $C_{D, \text { total }}$ & Improvement (\%) \\
\hline Baseline & 0.375 & 0.022 & 0.397 & - \\
Closed rectangular flap & 0.315 & 0.019 & 0.334 & 15.87 \\
Roof surface with cavity & 0.299 & 0.019 & 0.318 & 19.82 \\
Side rams & 0.286 & 0.020 & 0.307 & 22.67 \\
\hline
\end{tabular}

\section{CONCLUSION}

A three-dimensional SUV was designed in CATIA, and a numerical flow simulation was conducted using AnsysFluent. The mesh and the physical model used in the flow simulation were validated using existing experimental data, and excellent agreements were obtained.

i. A closed rectangular flap as an add-on device was used to restructure the wake vortex system. Results reveal a stretched wake compared to that of the baseline case. Modification in the vortex system topology enhances the merging and decay of the vehicle wake vortex system. A gradual increase of base pressure towards the closedrectangular flap ends results in a drag reduction of $15.87 \%$.

ii. The perforated roof surface layer generates local vorticity and delays flow separation with higher base pressure values. This increase was reflected in a net drag reduction of $19.82 \%$.

iii. Air guided through carefully designed side rams introduces steady blowing close to the vortex cores leads to a confined wake area. The net effects appear in a pronounced drag reduction of $22.67 \%$.

\section{ACKNOWLEDGEMENT}

This work is supported by the International University of Rabat (UIR). We are grateful to the LERMA laboratory for providing the In-house computing resources.

\section{REFERENCES}

[1] R. M. Wood. "Impact of advanced aerodynamic technology on transportation energy consumption," SAE Transact., vol. 113, pp. 854-874, 2004, doi: 10.4271/2004-01-1306.

[2] J. C. Lin, J. Towfighi, and D. Rockwell. "Near-Wake of a circular cylinder: Control, by steady and unsteady surface injection," J. Fluids Struct, vol. 9, no. 6, pp. 659-669, 1995, doi: 10.1006/jfls.1995.1036. 
[3] A Brunn and W Nitsche. "Active control of turbulent separated flows over slanted surfaces," Int. J. Heat Fluid Flow, vol. 27, no. 5, pp. 748-755, 2006, doi: 10.1016/j.ijheatfluidflow.2006.03.006.

[4] J. Wang and L. Feng. Flow control techniques and applications, 2018.

[5] A. Altaf, A. A. Omar, and W. Asrar. "Review of passive drag reduction techniques for bluff road vehicles," IIUM Eng. J, vol. 15, no. 1 SE-Articles, pp. 61-69, 2014, doi: 10.31436/iiumej.v15i1.477.

[6] T Lee and L S Ko. "PIV investigation of flowfield behind perforated Gurney-type flaps," Exp. Fluids, vol. 46, no. 6, pp. 1005-1019, 2009, doi: 10.1007/s00348-008-0606-1.

[7] A. Altaf, A. A. Omar, and W. Asrar. "Passive drag reduction of square back road vehicles," J. Wind Eng. Ind. Aerodyn, vol. 134, pp. 30-43, 2014, doi: 10.1016/j.jweia.2014.08.006.

[8] J. F. Beaudoin and J. L. Aider. "Drag and lift reduction of a 3D bluff body using flaps," Exp. Fluids, vol. 44, no. 4, pp. 491501, 2008, doi: 10.1007/s00348-007-0392-1.

[9] J. Ha, S. Jeong, and S. Obayashi. "Drag reduction of a pickup truck by a rear downward flap," Int. J. Automot. Technol., vol. 12, no. 3, pp. 369-374, Jun. 2011, doi: 10.1007/s12239-011-0043-7.

[10] J. J. Kim et al., "Salient drag reduction of a heavy vehicle using modified cab-roof fairings," J. Wind Eng. Ind. Aerodyn, vol. 164, pp. 138-151, 2017, doi: 10.1016/j.jweia.2017.02.015.

[11] J. Tian et al., "Aerodynamic drag reduction and flow control of Ahmed body with flaps,” Adv. Mech. Eng, vol. 9, no. 7, pp. 1-17, 2017, doi: 10.1177/1687814017711390.

[12] A. R Paul, A. Jain, and F. Alam. "Drag reduction of a passenger car using flow control techniques," Int. J. Automot. Technol., vol. 20, no. 2, pp. 397-410, 2019, doi: 10.1007/s12239-019-0039-2.

[13] A. Mariotti, G. Buresti, and M. V. Salvetti. "Separation delay through contoured transverse grooves on a 2D boat-tailed bluff body: Effects on drag reduction and wake flow features," Eur. J. Mech. B/Fluids, vol. 74, pp. 351-362, 2019, doi: 10.1016/j.euromechflu.2018.09.009.

[14] S. Sadeghipour et al., "Control of flows around bluff bodies mediated by porous materials," Exp. Therm. Fluid Sci, vol. 114, pp. 1-14, 2020, doi: 10.1016/j.expthermflusci.2020.110048.

[15] M. Lorite-Díez et al.,"Drag reduction on a three-dimensional blunt body with different rear cavities under cross-wind conditions." J. Wind Eng. Ind. Aerodyn, vol. 200, pp. 1-11, May 2020, doi: 10.1016/j.jweia.2020.104145.

[16] J. Mode. "Simulation of the flow over a flat dimpled plate," Available: https://repository.asu.edu/attachments/56200/ content/Mode_asu_0010N_10158.pdf. 2010 (accessed: Jun. 28, 2021).

[17] S. F. Wong and S. S. Dol. "Simulation study on vehicle drag reduction by surface dimples," publications.waset.org, Available: http://publications.waset.org/10003966/simulation-study-on-vehicle-drag-reduction-by-surface-dimples. (accessed: Jun. 28, 2021).

[18] K. Kumar Shaw, Y. Kesarwani, and P. Chakravarty. "Study of dimple effect on aerodynamic drag characteristics of a car," Prepr. Int J Innov Res Sci Eng Technol, vol. 9, pp. 4628- 4637, 2020, doi: 10.13140/RG.2.2.28628.42888.

[19] A. Brunn et al., "Active drag control for a generic car model,” in Notes Numer. Fluid Mech. Multidiscip. Des., vol. 95, pp. 247-259, 2007, doi: 10.1007/978-3-540-71439-2_15.

[20] T. Heinemann et al., "Active flow control on a 1:4 car model," Exp. Fluids, vol. 55, no. 5, pp. 1-11, 2014, doi: 10.1007/s00348-014-1738-0.

[21] R. P. Littlewood and M. A. Passmore. "Aerodynamic drag reduction of a simplified squareback vehicle using steady blowing," Exp. Fluids, vol. 53, no. 2, pp. 519-529, 2012, doi: 10.1007/s00348-012-1306-4.

[22] S. Aubrun et al., "Separation flow control on a generic ground vehicle using steady microjet arrays," Exp. Fluids, vol. 51, no. 5, pp. 1177-1187, 2011, doi: 10.1007/s00348-011-1132-0.

[23] B. F. Zhang et al., "Active drag reduction of a high-drag Ahmed body based on steady blowing,” J. Fluid Mech, vol. 856, pp. 351-396, 2018, doi: 10.1017/jfm.2018.703.

[24] J. J. Cerutti et al., "Active flow control on a square-back road vehicle," Fluids, vol. 5, no. 2, pp. 1-27, 2020, doi: 10.3390/fluids5020055.

[25] H. Harinaldi, B. Budiarso, and S. Pangihutan Simanungkalit. "Effect of active control by blowing to aerodynamic drag of bluff body van model utilization of natural iron ore for catalytic reaction of (pyroligneous acid) derived from palm kernel shells view project," Int. J. Fluid Mech. Res., 2013, doi: 10.1615/InterJFluidMechRes.v40.i4.

[26] H. Harinaldi, B. Budiarso and, S. Pangihutan Simanungkalit. "Computational analysis of active control to reduce aerodynamics drag on a van model Synjet-heat View project Turbulent flow control-mass transfer View project," 2011. Available: https://www.researchgate.net/publication/268809627 (accessed: Jun. 25, 2021).

[27] M. Jahanmiri and M. Abbaspour. "Experimental investigation of drag reduction on Ahmed model using a combination of active flow control methods," Int. J. Eng. Trans. A: Basics, vol. 24, no. 4, pp. 403-410, 2011, doi: 10.5829/idosi.ije.2011.24.04a.09.

[28] S. W. Baek and S. W. Lee. "Aerodynamic drag reduction on a realistic vehicle using continuous blowing," Microsyst. Technol., vol. 26, no. 1, pp. 11-23, 2020, doi: 10.1007/s00542-019-04355-w.

[29] J. Mosiężny et al., "Base drag reduction concept for commercial road vehicles," Energy, vol. 205, pp. 1-15, 2020, doi: 10.1016/j.energy.2020.118075. 
[30] G Fourrié et al., "Bluff-body drag reduction using a deflector," Exp. Fluids, vol. 50, no. 2, pp. 385-395, Feb. 2011, doi: 10.1007/s00348-010-0937-6.

[31] A. Chainani and N. Perera. "CFD investigation of airflow on a model radio control race car," 2008. Available: http://nrl.northumbria.ac.uk/235/ (accessed: Jun. 25, 2021).

[32] W. H. Hucho and G. Sovran. "Aerodynamics of road vehicles,” Annu. Rev. Fluid Mech, vol. 25, no. 1, pp. 485-537, 1993, doi: 10.1146/annurev.fl.25.010193.002413.

[33] S. R. Ahmed, G. Ramm, and G. Faltin. "Some salient features of the time-averaged ground vehicle wake." SAE Trans., Tech. Pap., No. 840300. pp. 473-503, 1984, doi: 10.4271/840300.

[34] ANSYS Fluent Theory Guide; Ansys. Inc.: Canonsburg, PA, USA, 2020.

[35] U. Goldberg et al., "The k-e-Rt turbulence closure," Eng. Appl. Comput. Fluid Mech., vol. 3, no. 2, pp. 175-83, 2009, doi:10.1080/19942060.2009.11015263.

[36] C. Hinterberger, M. García-Villalba, and W. Rodi, Large eddy simulation of flow around the Ahmed body. Berlin: Springer, 2004. 